\title{
Expérience de mise en dépôt des sédiments de dragage dans une ancienne ballastière
}

\author{
Sandrine Samson, Antoine Delouis ${ }^{1}$ \\ Jean-Marc Bérépion ${ }^{2}$
}

Résumé :

La gestion des sédiments de dragage nécessite aujourd'hui la recherche de solutions nouvelles, parmi lesquelles la mise en dépôt dans d'anciennes ballastières. Des études approfondies sur l'incidence de dépôts de sédiments, et principalement sur les aspects hydrogéologiques ont été développées. A la suite des expertises favorables, un site expérimental a été équipé. L'expérience donne lieu à un suivi des paramètres du milieu à l'aide de stations automatiques de mesures en continu et de prélèvements multiples. Deux ans après le début de cette nouvelle pratique, des premiers enseignements peuvent être dégagés.

\section{Abstract :}

The dredging sediment management needs today to look for new solutions, among which the deposit in former gravel quarries, now transformed in ponds. Elaborate studies about the impact of underwater sediment deposit, and mostly on hydrogeological aspects, have been developped. After the agreement given by the experts, an experimental deposit site has been fitted. The experiment is controlled by tracking several environmental parameters with real-time automatic measurement stations and multiple samplings. Two years after the beginning of this new practice, first conclusions can be reached.

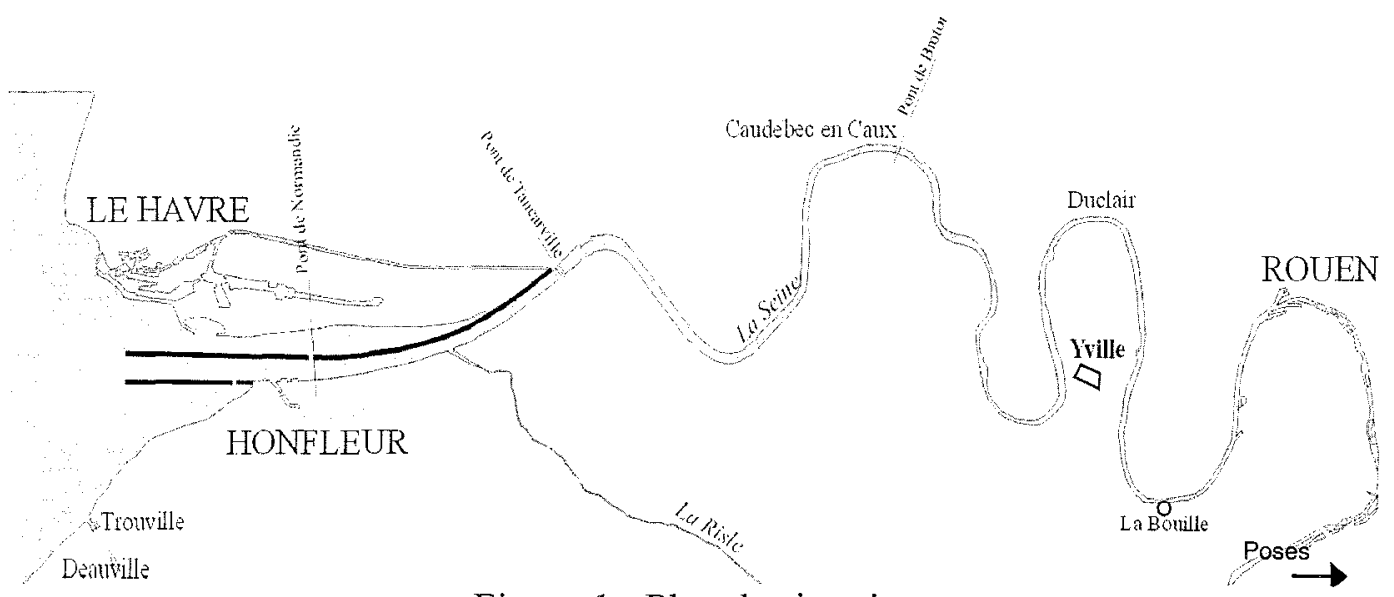

Figure 1 : Plan de situation

\footnotetext{
${ }^{1}$ Service de l'Environnement Maritime et du Chenal - Port Autonome de ROUEN, 34 bd de Boisguilbert BP 4075, 76022 ROUEN CEDEX (tél : 02.35.52.54.49 sec@rouen.port.fr)

${ }^{2}$ Directeur du Parc Naturel Régional des Boucles de la Seine Normande, 76940 Notre Dame de Bliquetuit, (tél : 02.35.37.23.16 jberepion@yahoo.fr)
} 


\section{Introduction : les dragages à Rouen}

Le Port Autonome de Rouen est situé sur l'estuaire de la Seine, à 120 kilomètres de la mer, au plus loin dans les terres; c'est le port maritime de la région parisienne avec ses 20 millions d'habitants et $40 \%$ de l'industrie française dans un rayon de $200 \mathrm{~km}$.

Comme pour l'ensemble des ports maritimes, qu'ils soient côtiers ou situés en fond d'estuaire, le maintien des caractéristiques du chenal de navigation impose des dragages d'entretien réguliers. Ceux-ci peuvent être décrits sommairement par les 3 domaines suivants :

- l'estuaire aval, dans sa partie haline, se trouve aujourd'hui dans un quasi équilibre morphologique du fait de l'arrêt des travaux structurants depuis 1980 . Mais cet équilibre reste très fragile. Le chenal en aval de Tancarville connaît deux points singuliers sur le plan sédimentologique; le débouché des digues submersibles où il traverse la barre d'embouchure de l'estuaire, et, à la hauteur $\mathrm{du}$ pont de Normandie, la brèche dans la digue basse nord qui constitue une zone de perturbation des courants de flot (apports par la zone nord) et de dissipation partielle des courants de jusant. On constate sur ces deux secteurs $\mathrm{du}$ chenal une sédimentation très active. Les sédiments à extraire sont très majoritairement des sables, provenant de la mer, transportés par le courant du Calvados, et mis en circulation dans l'estuaire sous l'action des houles et des courants alternatifs des marées. Les dragages concernent environ 4 millions de tonnes de matières sèches, par an, avec des fluctuations.

- la partie du chenal entre la Bouille et Tancarville, soit environ 100 kilomètres, est soumise aux forts courants de marées, de telle sorte que les sédiments ne s'y déposent pas de façon pérenne, excepté dans la partie concave de certaines courbes du fleuve. Répartis en 5 « bancs » principaux, les sédiments à draguer sont composés de sables et/ou de limons, pour environ 60000 tonnes chaque année.

- la zone portuaire amont, de Rouen à la Bouille, est, du fait des élargissements que constituent les zones d'évitage, les bassins et les sur-approfondissements que sont les souilles de quai, le siège d'une sédimentation intense. Les sédiments apportés par le fleuve, principalement lors des crues, s'y déposent ; si les apports du fleuve à Poses peuvent être estimés, en moyenne annuelle, à environ 600000 tonnes, ce sont environ 250000 tonnes de limons par an qui nécessitent d'être dragués. Une campagne est organisée au printemps, après les crues, pour les deux tiers environ des volumes annuels. Une seconde a lieu en fin d'étiage.

Ces dragages sont opérés à l'aide de deux dragues aspiratrices en marche; la «Paul Barrillon », de forte capacité (puits de $4000 \mathrm{~m}^{3}$ ), est affectée à l'estuaire, la « Ronceray », plus modeste (puits de $1500 \mathrm{~m}^{3}$ ) se concentre sur les secteurs amont. 


\section{Devenir des sédiments draqués}

Si les sédiments extraits dans la partie maritime sont "clapés » en mer, les sédiments extraits dans les zones amont, compte tenu de l'impact des distances de transport sur l'économie des opérations, sont mis «à terre », et donc sortis du milieu fluvial. Traditionnellement, ces sédiments sont mis dans des chambres de dépôt, enceintes fermées par des digues, dans lesquelles les sédiments sont refoulés hydrauliquement, puis, pour les matériaux fins, se consolident à l'air. Le passage d'engins spéciaux permet d'augmenter les vitesses de consolidation en favorisant l'évapo-transpiration.

Les importantes quantités draguées annuellement conduisent à créer des « tas » en bord de Seine. Parallèlement, les extracteurs des granulats ont excavé de nombreuses ballastières, des «trous", dans les boucles de la Seine. Ces deux activités sont consommatrices de zones humides qui caractérisent le lit majeur du fleuve. L'avenir respectif des deux problématiques de développement a donc conduit à l'interpellation: "mettre les tas dans les trous». Cette action est conforme à l'esprit d'expérience et d'actions exemplaires à développer au sein d'un Parc Naturel Régional

Cette idée a été inscrite dans le cadre du schéma départemental des carrières comme mode de réaménagement principal pour l'ouverture de nouvelles exploitations, ainsi que dans la perspective du réaménagement de ces espaces avec une qualité environnementale favorable notamment à l'avifaune, par la création de prairies et de zones humides.

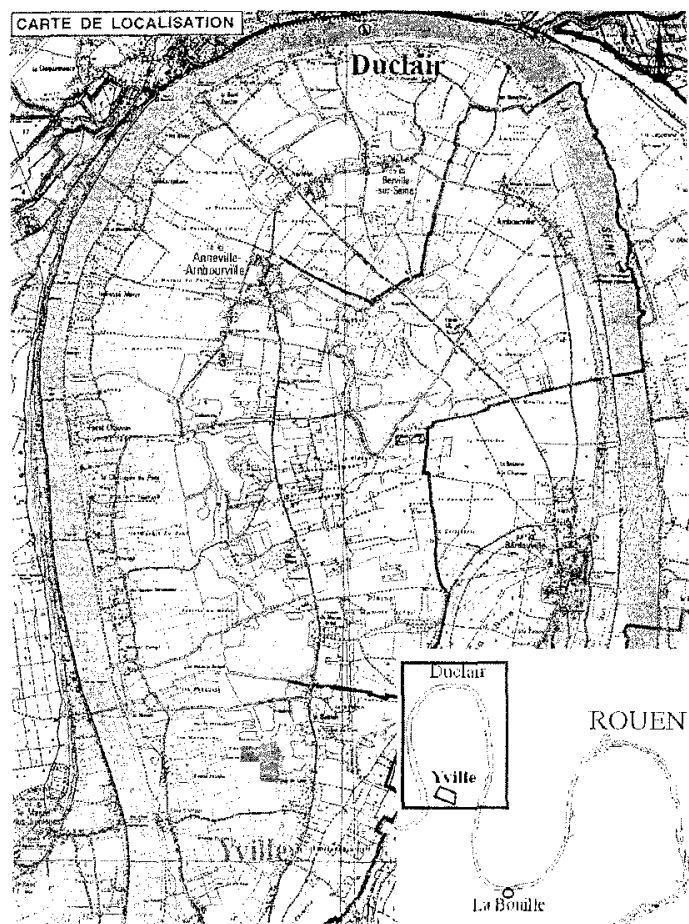

Ce mode de gestion des sédiments de dragage représente une alternative au stockage traditionnel en chambre de dépôt à terre de ces sédiments, en pouvant à priori accueillir environ $75 \%$ des volumes de dragages d'entretien de la zone portuaire. Cette possibilité de gestion, si elle s'avère concluante, permettra une réduction très importante de la demande de terrains pour la gestion des sédiments.

Elle représente donc un élément déterminant pour la construction d'un schéma directeur de gestion des sédiments de dragage, dont une autre voie, complémentaire, sera la possibilité de valorisation et de réutilisation des sédiments.

Figure 2 : Plan de localisation d'Yville-sur-Seine 


\section{L'expérience de remblaiement d'une ancienne ballastière}

L'opération pilote consiste donc à remblayer, avec des sédiments de dragage d'entretien de la zone portuaire amont, une ancienne ballastière d'un volume de l'ordre de $1000000 \mathrm{~m}^{3}$ à Yville-sur-Seine, dans la boucle d'Anneville Ambourville.

Cette opération revêt un caractère expérimental novateur : en effet, il n'existe pas, à notre connaissance et celle des experts consultés, d'exemples similaires dont on puisse tirer les enseignements suffisants pour une exploitation directe sans vérifications et études de terrain. Pour cela un programme de suivi scientifique a été mis en place avec l'appui des Services de l'Etat et les experts pour tirer toutes les conclusions nécessaires à la recevabilité de ce mode de gestion et organiser sa généralisation dans une perspective à long terme.

\subsection{Spécificité des équipements de l'opération d'Yville-sur-Seine}

Dans le cadre des chambres de dépôt traditionnelles, les équipements comprennent un engin de dragage mécanique associé à des chalands porteurs pour assurer le transfert des sédiments vers le lieu de mise à terre, et un engin nautique de refoulement hydraulique qui permet, via une conduite, de rejoindre la chambre disposée en bord de fleuve. L'expérience de remblaiement de la ballastière d'Yville-sur-Seine a nécessité de réétudier l'ensemble des équipements.

En premier lieu l'engin de dragage mécanique (drague à godets) et sa desserte de chalands ont été remplacés par une drague aspiratrice en marche auto porteuse. Il faut donc prévoir une infrastructure d'accostage adaptée, au plus proche du site de l'expérimentation.

La liaison entre la drague et la colonne de refoulement doit tenir compte des distances entre le fleuve et la ballastière, soit environ $1200 \mathrm{~m}$. Il y a lieu de dimensionner correctement l'ensemble des dispositifs de pompage et de transport des sédiments; il s'est avéré nécessaire de mettre en cuvre une station de pompage relais pour augmenter les capacités de transport, station à installer sur un ponton aménagé pour accueillir l'organe de transfert, le booster et sa motorisation.

Il faut également mettre en place une conduite hydraulique, de diamètre réduit par rapport aux conduites usuelles $(500 \mathrm{~mm}$ au lieu de $600 \mathrm{~mm}$ ), fonction des pertes de charges sur la longueur de transfert des sédiments qui se révèle dans l'espèce plus que doublée par rapport au cas des chambres de dépôts à terre.

Les sédiments transportés dans une colonne à haute vitesse, sous la forme d'une mixture constituée d'environ $90 \%$ d'eau, sont déversés après dissipation de la vitesse, pour s'écouler sous forme de courant de turbidité sur les fonds de la ballastière. 
Enfin, des dispositions de conception entièrement nouvelles pour les dispositifs spécifiques d'introduction des sédiments dans le plan d'eau ont été mis en œuvre. Une cheminée de dissipation d'énergie permet de réduire la vitesse d'introduction de la mixture dans le plan d'eau afin de favoriser le développement de courants de turbidité sur les fonds de la ballastière, en évitant l'explosion de ce mélange eausédiment en surface du plan d'eau.

Des pompes assurent un retour vers la Seine des eaux excédentaires ; par mesure de précaution, le niveau du plan d'eau est contrôlé automatiquement pour éviter tout débordement. Il est mis en dépression par rapport à la nappe environnante avant les refoulements, de façon à inverser les gradients hydrauliques en tant que de besoin, et prévenir ainsi des risques de transfert éventuel vers la nappe environnante.

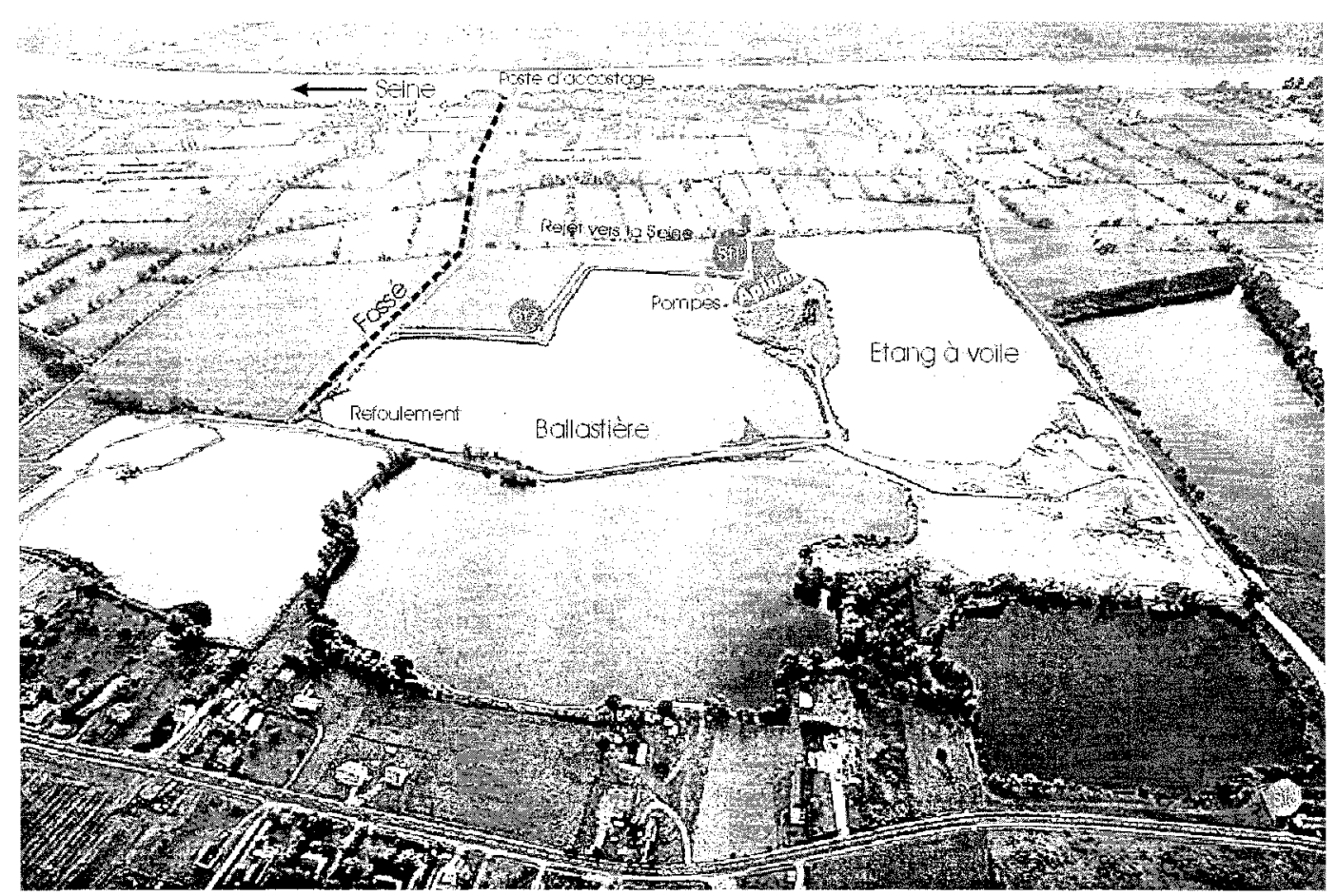

Figure $n^{\circ} 3$ : la ballastière d'Yville et son équipement

\subsection{Mesures d'accompagnement environnemental}

Il s'agit, dans le cadre d'un suivi organisé autour d'un comité scientifique, d'analyser les effets du comblement de la ballastière sur l'hydraulique et l'hydrogéologie du site, ainsi que sur les écosystèmes.

L'objet des dispositifs physiques mis en œuvre consiste donc en :

- des mesures spécifiques à l'expérimentation, nécessaires à la validation de ce type de gestion alternative. 
- l'enrichissement de la connaissance du milieu, notamment en matière d'hydrogéologie, de façon à mettre en œuvre ultérieurement des outils d'aides à la décision pour l'établissement d'un schéma généralisant ce type d'opération dans les boucles similaires du bief maritime de la vallée de la Seine.

- des mesures et analyses pour l'évaluation de la capacité de reconstitution de milieux humides dans les boucles.

Pour cela, il y a lieu de prévoir des stations de mesures en continu et des campagnes de prélèvements pour suivre et valider cette expérience, de façon à constituer un réseau d'observation de la qualité des eaux superficielles et souterraines dans l'environnement de l'expérience et de son champ d'application potentiel.

Des dispositions particulières sont également prises en compte pour l'évaluation et l'orientation des procédés mis en œuvre, de façon à en améliorer les conditions d'exécution. Citons pour exemple les dispositifs de contrôle et de gestion des niveaux dans la ballastière et la nappe environnante en cours de remblayage, décrits précédemment dans leur principe.

Des études spécifiques à la généralisation de cette opération sont prévues pour étudier, riches des résultats de ce réseau d'observation, les meilleures orientations et dispositions constructives pour une généralisation, permettant alors de gérer à long terme les sédiments de dragage.

Des mesures collatérales de préservation d'une plante protégée identifiée sur le site, Apium Repens, et des expériences de création d'habitats favorables sont mises en œuvre. Il s'agit également de développer une meilleure connaissance des sensibilités du milieu pour la reconstruction la plus adaptée de zones humides sur les terrains concernés aujourd'hui et demain par ces travaux.

\subsection{Eléments issus des études préliminaires}

Les études sur le comportement hydrogéologique de la boucle en général et de la ballastière dans son environnement proche en particulier montrent que :

- le fond de la ballastière est colmaté par un tapis de fines de lavage des granulats résultant de l'extraction, les parois latérales ne le sont que partiellement, les vitesses sont extrêmement faibles : les débits d'échange sont de l'ordre de $50 \mathrm{~m}^{3} / \mathrm{h}$ pour un linéaire de $800 \mathrm{~m}$ et une profondeur utile de l'ordre de $6 \mathrm{~m}$.

- les ballastières se comportent comme un drain vis-à-vis de la nappe dans la plaine, et comme un amortisseur-tampon vis-à-vis de la marée ; si la marée se manifeste encore à plus de $1000 \mathrm{~m}$ de la Seine dans la nappe, seuls les cycles de vives eaux - mortes eaux sont lisibles dans la ballastière. Les sédiments 
déposés ont une perméabilité très faible et constituent une masse semiimperméable, pouvant modifier les écoulements dans la nappe et la piézométrie.

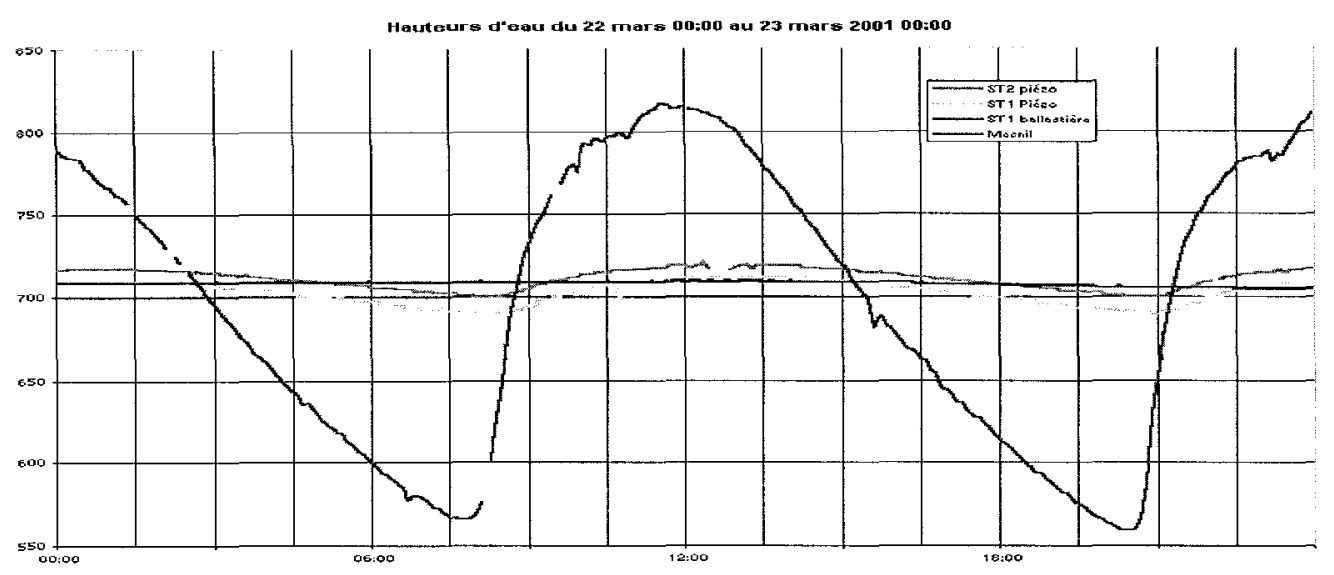

Figures $n^{\circ} 4$ et 5 : Evolution des niveaux piézométriques

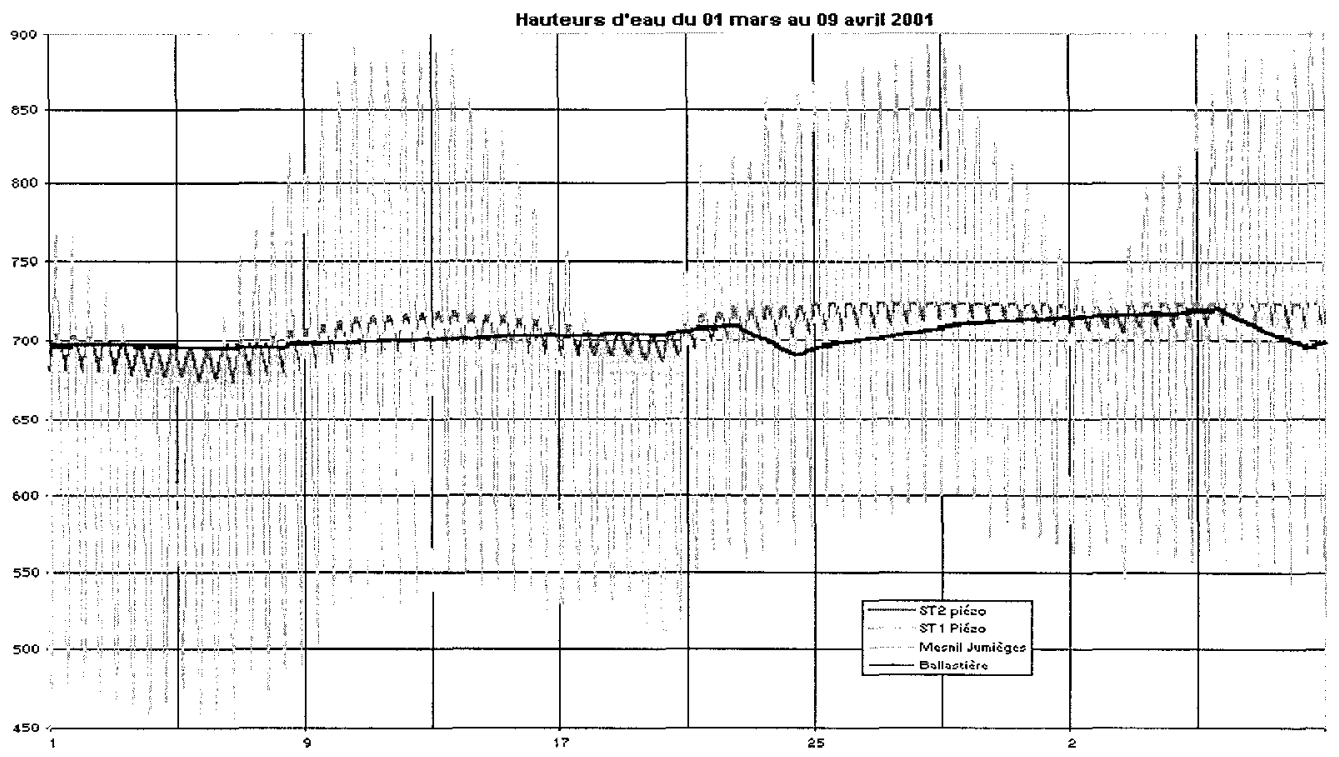

- les sédiments sont porteurs d'une charge anthropique mais ils ne montrent pas de charge dans les lixiviats : une grille de valeurs de référence pour l'appréciation de la qualité chimique ainsi que des critères d'acceptabilité dans la ballastière au travers des teneurs ont été établis. De plus, jusqu'à présent tous les sédiments ont été jugés recevables.

- des tests d'écotoxicité, réalisés par l'INERIS ${ }^{3}$, sur les organismes vivants, benthiques et pélagiques, et sur les végétaux aquatiques n'ont montré aucune toxicité, ni létale, ni chronique. D'autres tests sur des végétaux et animaux terrestres n'ont rien montré non plus.

\footnotetext{
${ }^{3}$ INERIS : Institut National de l'Environnement industriel et des RISques
} 


\subsection{Premiers résultats et premiers enseignements}

L'expérience a fait l'objet d'un arrêté préfectoral d'autorisation au titre de la loi sur l'eau début 1999. L'été de cette année là a été mis à profit pour la construction de l'infrastructure d'accostage, la création de la piste d'accès dans le marais, le montage des colonnes et de l'ensemble des équipements. La première campagne de déversement a eu lieu au printemps 2000, et nous pouvons donc aujourd'hui tirer des résultats au vu de 4 campagnes de refoulement sur une durée de 2 années complètes, pour un volume remblayé supérieur à $250000 \mathrm{~m}^{3}$.

Les eaux de la ballastière, celles de la nappe, et les eaux de Seine qui permettent le transport hydraulique des sédiments présentent des caractéristiques très différentes. Tout mélange des mixtures et des eaux de transport doit donc se manifester de façon nette sur les paramètres globaux.

Les suivis de paramètres en continu dans la nappe à proximité immédiate de la ballastière ne montrent aucune modification du milieu. Sont mesurés le $\mathrm{pH}$, la turbidité, l'oxygène dissous et la conductivité électrique :

- le pH de l'eau de la ballastière a progressivement cru, du fait de l'introduction de l'eau de Seine. Dans la nappe, on n'observe pas d'évolution, l'eau restant légèrement acide.

- l'oxygène dissous, quasi absent dans la nappe, n'évolue pas alors que l'eau de la ballastière et l'eau de la Seine présentent des teneurs significatives.

- la turbidité est la mesure la plus délicate, dans des piézomètres où les circulations restent faibles, et les capteurs très fragiles : pas d'enseignement particulier à tirer de cette mesure.

- la conductivité électrique est le paramètre le plus approprié pour mettre en évidence des échanges entre la ballastière et la nappe, notamment en ce qui concerne les polluants métalliques: une relation entre le plan d'eau et la nappe se traduirait immédiatement par une baisse significative de la conductivité. En effet, la ballastière présente une conductivité de l'ordre de $400 \mu \mathrm{S} / \mathrm{cm}$, l'eau de Seine environ $600 \mu \mathrm{S} / \mathrm{cm}$, l'eau de la nappe 1000 à $1100 \mu \mathrm{S} / \mathrm{cm}$. La conductivité de la ballastière a très légèrement crû, celle de la nappe n'a pas évolué du tout. 


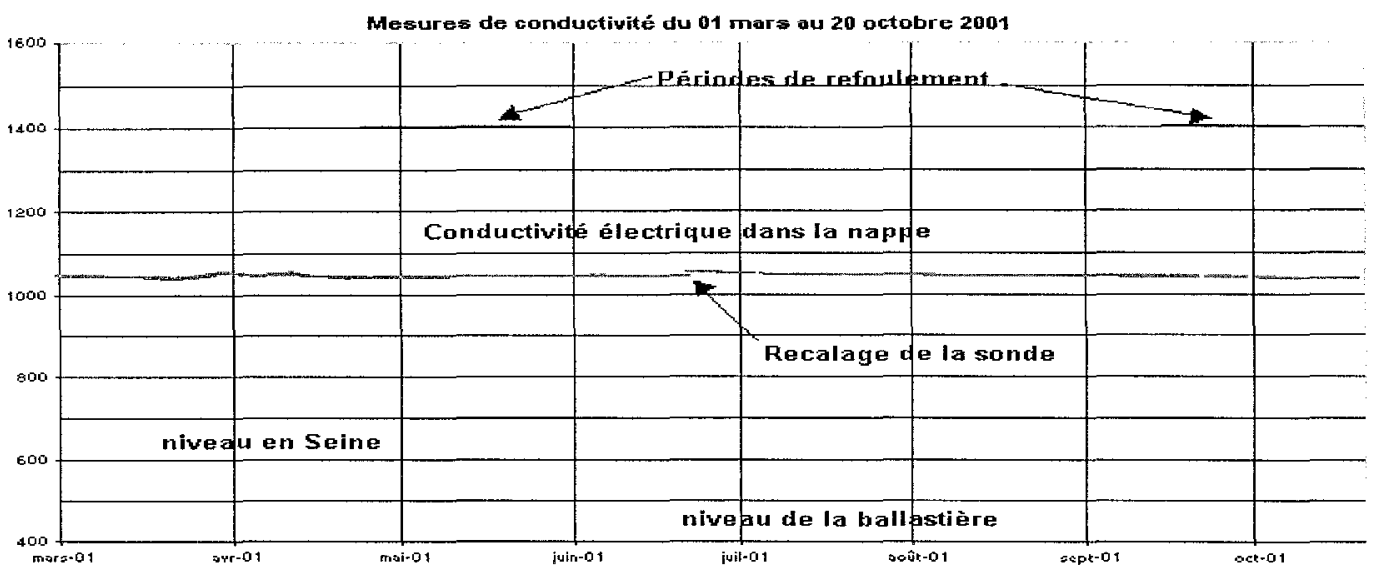

Figure $n^{\circ} 6$ : Evolution de la conductivité électrique

Les analyses régulières par prélèvement, dans les piézomètres où sont installées les sondes en continu, dans les différents plans d'eau et dans les fossés, ne montrent aucune altération de la qualité chimique de l'eau.

Il n'apparaît aucun transfert de contaminant de la ballastière vers la nappe.

Les poissons pêchés à l'automne 2001 dans la ballastière ne montrent ni mortalité par asphyxie ou empoisonnement, ni stress particulier, même pour des espèces réputées sensibles comme la perche.

Enfin, la plante protégée, Apium Repens, a donné lieu à des travaux de génie écologique. Il a été reconstitué des habitats favorables pour cette plante exigeante en terme d'hygrophilie et d'ouverture des milieux. L'expérience au bout de deux ans montre une recolonisation de cette espèce, mais dans la fragilité de l'évolution d'un milieu où des plantes ubiquistes ont une fâcheuse tendance à occuper le terrain. Une gestion appropriée est mise en œuvre, par fauches régulières d'abord, et par pâturage extensif ensuite.

\section{Conclusions}

Ce nouveau mode de gestion a posé de très nombreuses interrogations avant d'être mis en œuvre. Les aspects hydrogéologiques ont été les premiers lieux de réflexion et d'études approfondies; jusqu'à présent, les expertises confiantes sur la faiblesse d'un risque de transfert, associées aux mesures de précaution par l'inversion des gradients hydrauliques, se sont traduites dans les faits par l'absence de transfert vers la nappe.

La qualité écologique des espaces reconstitués par ces remblaiements fera l'objet d'une deuxième étape d'expérience et de suivi, les années à venir. 
La réussite de cette expérience ouvre le champ à un mode de gestion des sédiments de dragage plus économe d'espaces dans ce territoire exigu et riche qu'est la vallée de la Seine; elle donne de nouvelles perspectives à la réhabilitation nécessaire des ballastières qui trouvent ainsi une recomposition environnementale inattendue, témoignant d'une synergie entre deux activités économiques (carrières et ports)

Cette opération a été réalisée avec le concours du Fonds Européen de Développement Régional, de l'Agence de l'Eau Seine-Normandie, le soutien du Parc Naturel Régional des Boucles de la Seine Normande, et la bonne volonté de tous, représentants de la Commune d'Yville-sur-Seine et en particulier de son Maire, représentants des Services de l'Etat, scientifiques au sein d'un comité d'évaluation préalable et d'un comité de suivi de l'expérience, associations locales de protection et de défense de l'environnement.

Bibliographie :

- La valorisation des produits de dragage ; A.I.P.C.N.,G.T.n ${ }^{\circ} 19$ du CTP II ; Supp $1^{t}$ bulletin 77

- Etude des impacts hydrauliques souterrains du projet de comblement de gravières par des produits de dragage ; SAFEGE 1995

- Etude d'impact écologique et hydrobiologique du projet de remblayage d'une ballastière ; ECOSPHERE 1998

- Inventaire floristique et propositions techniques de préservation de l'ache rampante sur le site d'Yville-sur-Seine ; ECOSPHERE 1998

- Projet expérimental de remblayage d'une ballastière par des sédiments de dragage de la Seine : étude d'impact et projet de réaménagement ; ENCEM 1998 